\title{
The Use of Web 2.0 Concepts to Support Deliberation in Spatial Decision-Making
}

\section{Claus Rinner}

Ryerson University

\section{Carsten Keßler}

University of Münster

\section{Stephen Andrulis}

Ryerson University

\section{digital.library.ryerson.ca/object/225}

Please Cite:

Rinner, C., Keßler, C., \& Andrulis, S. (2008). The use of Web 2.0 concepts to support deliberation in spatial decision-making. Computers, Environment and Urban Systems, 32(5), 386-395.

doi:10.1016/j.compenvurbsys.2008.08.004

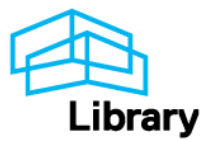




\title{
The Use of Web 2.0 Concepts to Support Deliberation in Spatial Decision-Making
}

\author{
Claus Rinner ${ }^{1 *}$, Carsten Keßler ${ }^{2}$, Stephen Andrulis ${ }^{1}$ \\ ${ }^{1}$ Department of Geography, Ryerson University \\ ${ }^{2}$ Institute for Geoinformatics, University of Münster \\ *corresponding author
}

\begin{abstract}
Technologies associated with the second-generation of the World-Wide Web enable virtually anyone to share their data, documents, observations, and opinions on the Internet. In less than three years, mapping platforms such as Google Maps have sparked an exponential growth in user-generated geographically referenced content. However, the "serious" applications of Web 2.0 are sparse and this paper assesses its use in the context of collaborative spatial decision-making. We present an online map-based discussion forum that enables Internet users to submit place-based comments and respond to contributions from other participants. We further use the geographic references in a thread-based master plan debate for a university campus to simulate this debate in the map-based forum. This allows us to demonstrate how the online map provides an overview of the status and spatial foci of the debate, and how it can help us understand the spatial thought processes of the participants.
\end{abstract}

Keywords: Analytic-Deliberative Approach, Argumentation Mapping, Participatory GIS, Spatial Decision Support Systems, Web 2.0

\section{Introduction}

Goodchild (2007a) posits the implementation of Gore's (1998) Digital Earth vision as the grand challenge for Geographic Information Science - a major research project that "has the ability to capture popular imagination” Goodchild (2007a, p. 605). Digital Earth is 
conceived as a "multi-resolution, three-dimensional representation of the planet, into which we can embed vast quantities of geo-referenced data" (Gore 1998) and as a tool for knowledge acquisition for everyone. Goodchild (2007a) also suggests using it as an “experimental environment” for planners. A recent addition to this vision is the contribution of information by digital earth users. User-generated content is a core feature of second-generation World-Wide Web applications subsumed under the label "Web 2.0" (O’Reilly 2005, Graham 2005). Within GIScience, Goodchild (2007b) uses the label "volunteered geographic information” for the contribution of local geospatial knowledge to community platforms.

To achieve sustainable development, local knowledge is increasingly being included in decision-making to complement traditional measurements through instruments and observation. When input from larger numbers and a broad range of community members is sought, the Internet is usually seen as an efficient medium for two-way communication between the general public and planners and decision-makers. Increasing numbers and diversity of stakeholders participating in spatial decision-making creates a growing potential for conflicting standpoints. The term “deliberation” (Stern and Feinberg (1996) will be used to denote discussion processes, arguing in favour or against decision alternatives, negotiation, and consensus-finding - methods that seek input from community members and take into account their preferences and opinions. Rinner (2005) discussed the role of argumentation to achieve sustainable development by integrating the objectives of diverse stakeholders.

Web 2.0 technologies provide a foundation for an exponentially growing amount of usergenerated content. Geotagging various media types (texts, photos, sound, animations) has become extremely popular. However, there is little evidence that the developers and providers of such applications are utilizing GIS research results such as spatial database concepts and principles of map design and collaborative geovisualization. For example, summaries of posted information are usually difficult or impossible to generate despite the fact that geographic references and spatial relations could be used to integrate and condense geotagged media. 
The remainder of this paper provides an overview of Web 2.0 concepts and techniques (section 2) and presents an argumentation mapping tool that is based on Google Maps (section 3). The tool is used to implement the geographic references in a master plan debate for a university campus (section 4). The results of the case study identify benefits and limitations of using Web 2.0 concepts to support deliberation in spatial decisionmaking (section 5). The paper concludes with an outlook on future research on spatial decision support through Web 2.0 (section 6).

\section{Web 2.0 and Deliberation in Spatial Decision-Making}

\subsection{User-Generated Content}

Various attempts have been made to characterize Web 2.0, with most of them using metaphors like the Web as a platform, the wisdom of the crowds, blogging, and social networks, all of which contribute a piece to the puzzle of what we call "Web 2.0" (O'Reilly 2005). In the context of this paper, we focus on two major shifts, those in user paradigms and in technology.

The shift in user paradigms is mainly characterized by the fact that users are no longer seen as mere consumers, as was the case both for classical media like newspapers, radio and television and the first-generation Web. User-generated content is now in the focus of a new industry and provides the basis for innovative services: users decide which news stories are interesting (digg.com), contribute to a free encyclopedia (Wikipedia), publish videos (YouTube) and share their expertise and personal opinions with readers of their blogs. Such content often has an implicit geospatial component, which increasingly is made explicit by adding geographic coordinates to the material's metadata (i.e. geotagging it). This way, the content can be visualized on a map and in some cases, the map material itself is user-generated content (OpenStreetMap 2007). The increasing volume and value of user generated geographic content has triggered an interest by GIScience researchers to study this phenomenon. Goodchild (2007b) uses the term “volunteered geographic information” for this variant of Web 2.0 content. 


\subsection{APIs, Web Services and AJAX}

A recent shift in Web technologies played a major role in facilitating the generation, online editing, and distribution of such contents. In contrast to the complex and expensive Web service architectures that were established for business applications, Web 2.0 services rely on lightweight application programming interfaces (APIs). These APIs are often easy-to-use and made publicly accessible for free, allowing programmers to combine services into so-called mashups (e.g. MapBuilder 2008, WikiMapia 2008, Twittervision 2007). The ease of use of Web 2.0 APIs stems from a combination of JavaScript for the actual programming functionality, and XML and the JavaScript Object Notation (JSON) as the preferred formats for data transfer. This combination, known as AJAX (Asynchronous JavaScript and XML), for the first time enables Web applications to behave more like desktop programs. In particular, users can now receive updated content from a Web site without having to wait for a reload of the entire page overcoming a usability issue that was apparent in the first-generation Web.

\subsection{Geographic Applications as Cornerstones of Web 2.0}

While traditional desktop GIS are among the most mature information systems, they were largely confined to professional users until Web 2.0 map applications made basic GIS functionality available to virtually anyone. Desktop GIS tend to be expensive and complex in use, and the required geospatial data are in most cases unaffordable for nonprofessionals. Open source solutions for Web mapping such as the UMN MapServer (MapServer 2008) or deegree (degree 2007) are often available for free, but require knowledge of digital maps, encodings and transfer protocols. In contrast, Web 2.0 applications such as Google Maps, Yahoo! Maps, or Microsoft Live Maps provide free access to easy-to-use functionality as well as high-quality map data.

These free services, all of which provide API access to programmers, have initiated an active community creating mashups. Additionally, new formats such as GeoRSS (GeoRSS 2008) for geotagging or the geo and adr microformats (Microformats 2008) for 
easy markup of spatial information on the Web have spurred a sharp increase in the nonprofessional use of online mapping tools. In contrast to traditional desktop GIS and the Web service interface specifications developed by the Open Geospatial Consortium (OGC), the functionality of Web 2.0 mapping APIs is limited. While this basic functionality might not be sufficient for many professional uses, the concentration on functionality that is relevant for lay users is the key to success for these services. Accordingly, recent developments have led to a split between GIS for professionals and “GIS for everyone”. By presenting a participatory GIS on the basis of Web 2.0, we attempt to combine professional GIS with broad-based inclusiveness.

\subsection{Participatory GIS and Deliberation in Spatial Decision-Making}

The term "participatory GIS" (PGIS) was coined in the mid-1990s in conjunction with a shift in focus from GIS technology and applications towards a critical evaluation of the uses of GIS in society (Harris et al. 1995). The variant "Public Participation GIS" emerged around the same time (Nyerges et al. 1997) and is usually associated with the use of GIS to foster grassroots involvement in policy decision-making (Sieber 2006). Stern and Fineberg (1996) introduced the notion of an analytic-deliberative approach to policy decision-making for situations with high decision equity such as risk management (Renn 1999). Jankowski and Nyerges (2003) translate the analytic-deliberative approach into a general framework for spatial decision-making.

Web 2.0 appears to provide the foundation for user-friendly online tools for collaborative spatial decision-making. AJAX-based user interfaces allow for seamless interaction with online applications. Publicly available geospatial data enable organizations to build PGIS with simplified or no licensing required. Moreover, important online mapping functions are pre-packaged in API toolboxes. From a user's perspective, the small number of service providers and the use of the same user interface and functionality in countless mashups facilitates recognition and learning. 
Despite these advantages, the use of Web 2.0 technology in collaborative spatial decision-making is still uncommon. Among numerous existing map mashups, we did not find any that are included formally in a planning process, let alone any that relate to the deliberative element in planning. To demonstrate the usefulness of the new technology at hand for PGIS, and to overcome the gap between research on collaborative spatial decision-making and recent developments in the realm of Web 2.0, we have implemented a Google Maps-based discussion forum called ArgooMap and demonstrate its usefulness in a case study application.

\section{A Web 2.0-based Argumentation Mapping Tool}

\subsection{Argumentation Maps: A Conceptual Model}

Rinner (2001) introduces argumentation maps as an object-based model for geographically referenced discussions. Argumentation maps provide the theoretical foundations for PGIS tools that support the deliberative aspects in spatial decisionmaking. More generally, the concept aims at supporting any argumentative process that has a spatial component and can benefit from explicit links between arguments and the corresponding places they refer to.

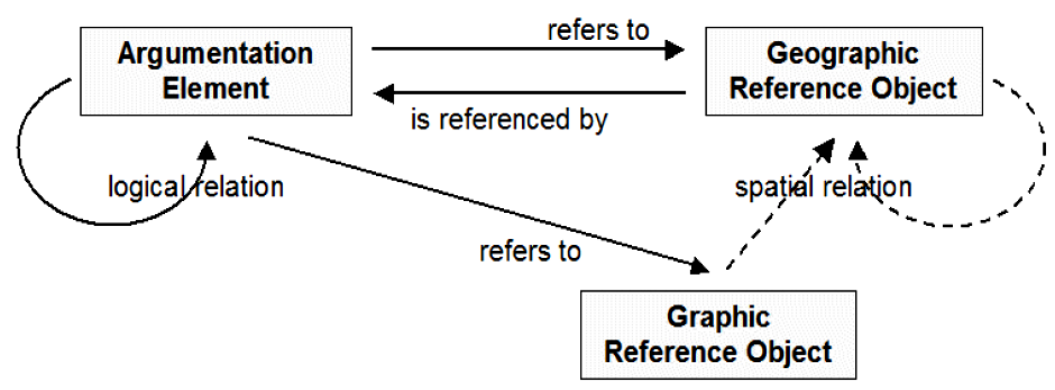

Figure 1: Conceptual model for argumentation maps (Source: Rinner 2006).

Rinner's (2006) argumentation map model defines argumentation elements and geographic reference objects as independent entities. From a user's point of view, it describes the relationships between a discussion and a map. The model also includes 
user-defined graphic reference objects. Thus, the model distinguishes between reference objects which are part of the map and reference objects which are created by users, e.g. to mark a point location or highlight an area. Between any kinds of objects in the model, many-to-many relationships are supported: an argument can refer to multiple (geo)graphic objects, a (geo)graphic object can be referenced by multiple arguments, an argument can be logically related to a number of other arguments, and (geo)graphic objects can be spatially related (see Figure 1).

The logical relations between arguments (e.g. a response to a suggestion, or a contra argument to a proposal) make a geographically referenced discussion more complex than a "flat" collection of geo-referenced media. On the one hand, there is an additional layer of complexity to be managed with respect to data storage, retrieval, and display, while on the other hand more advanced queries and analyses can be based on this model than on others. For example, the thread structure of a discussion suggests the display of message headings using indentation and enables both thread membership and location to be used as query filters.

\subsection{From Java to Google Maps-based Implementation}

A first argumentation maps implementation was developed based on Java Applets for the user interface and a combination of PHP and a MySQL database on the server side (Keßler et al. 2005a; Keßler et al. 2005b). It supported structured discussions with spatial reference objects as defined by the ArguMap model and was (to the best of our knowledge) the first tool to combine these two aspects in a single user interface (see Figure 2). This prototypical implementation has been used in a number of case studies (Sidlar and Rinner 2007, Rinner and Bird forthcoming, Simao et al. forthcoming), in which it was found to be useful for urban and regional planning scenarios, yet in some aspects difficult to understand for users unfamiliar with GIS functionality. Moreover, the implementation as a Java Applet (and Java WebStart in later versions) was often considered problematic, as it required the users to have a Java Virtual Machine installed. 
Based on the experience with this first version, and inspired by the first map mashups that appeared on the Web after the introduction of Google Maps, plans for a AJAX-based reimplementation emerged. The design and development of this second version was largely driven by the objective to improve the usability of the tool for lay users. Since the target group of a PGIS is the public, ease of use is essential for the success of such a system. The enhancements of Web applications as described above make the development of a powerful, yet accessible PGIS possible.

Among the available online mapping APIs, we decided to use the Google Maps API (Google 2008) for several reasons. The API was the most powerful in terms of functionality at the time we started the implementation, and there was already an active developer community using it, so that we could expect support and documentation. Moreover, there were already a number of Google Maps-based mashups in existence, so that a reasonable number of users could be expected to be familiar with this particular user interface.

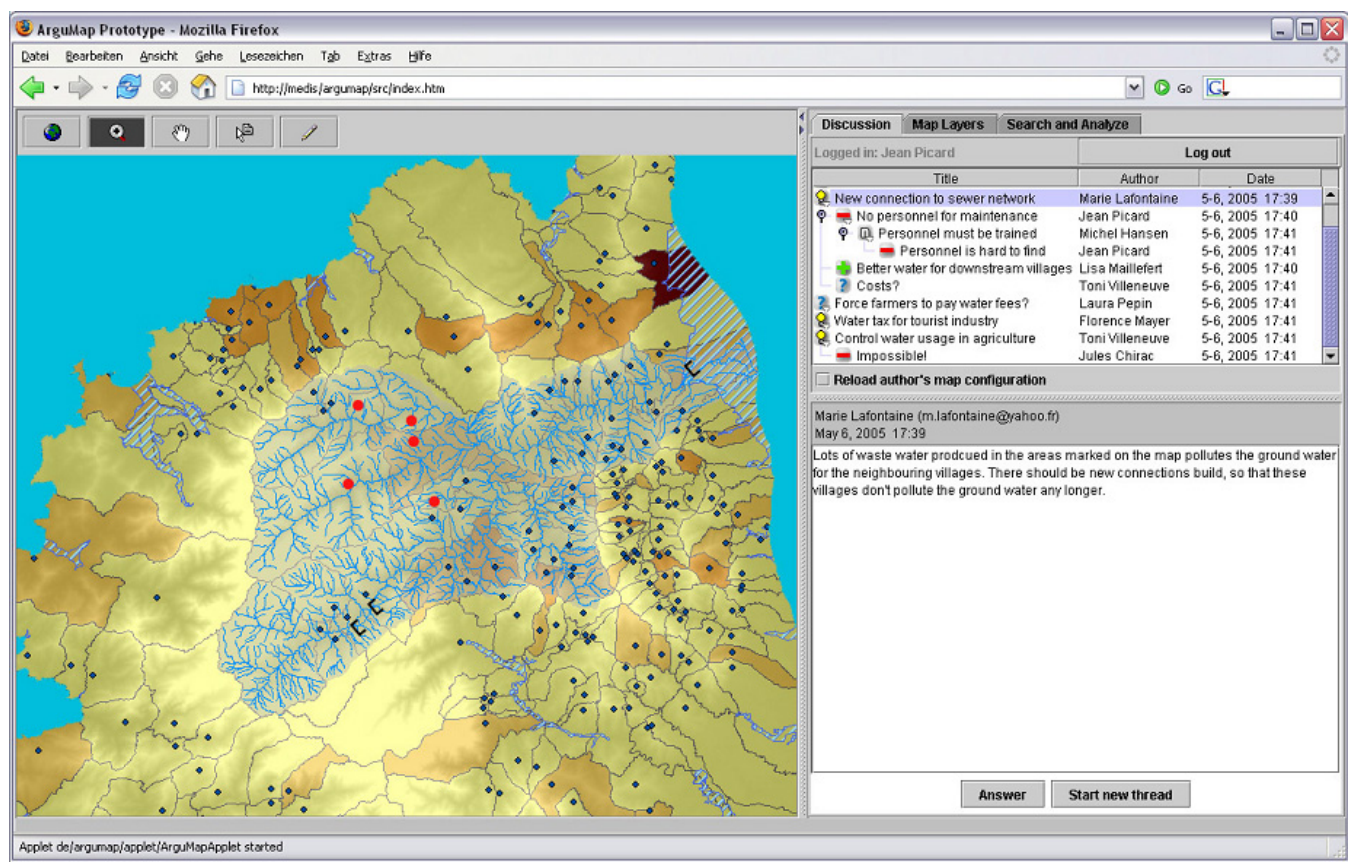

Figure 2: Screenshot of the first Java-based implementation of ArguMaps with a demo discussion relating to Mediterranean water resources (Source: Keßler et al. 2005b). 
Users in Rinner and Bird's (forthcoming) case study had problems understanding how discussion contributions and map elements were linked to each other, resulting in a large portion of arguments without explicit reference objects, although the contributions' texts contained explicit spatial references. Hence, the new version uses the map as an integrated interface for both the spatial references and the contributions to the discussion and does not show the discussion forum separately any more. This forces users to select at least one reference location for each new contribution to the discussion, with an option to add more references if necessary. The geographic reference objects are restricted to points in the current version, and users can either create new references by clicking on the map, or select existing references. Within the discussion, a contribution can only refer to (i.e. reply to or comment on) one other contribution. This is to keep the discussion easy to understand, but also to enable the display of the discussion as a tree. Nonetheless, each contribution can receive multiple replies.

Figure 3 provides an overview of the ArgooMap user interface and explains the different interaction modes. The figure shows the participant's initial view with indicators for places that are referenced by contributions to the discussion (top). A click on a marker opens a tree-structured overview of the contributions referencing this marker (bottom left). Clicking a message heading opens the display of a single discussion contribution, with the referenced markers highlighted on the map (bottom centre). The icons at the bottom of the pop-up window allow the user to jump directly to the answers for this contribution (down arrows), to write a reply (curved arrow), or to go back to the list of contributions for this marker (lines icon). Roll-overs provide explanations of the functionality behind the icons. A click on the reply icon or on an empty area on the map will open the input form for new contributions (bottom right). The first selected or created marker is green as the root marker for this contribution, additional markers can be selected or created and are highlighted in red. 


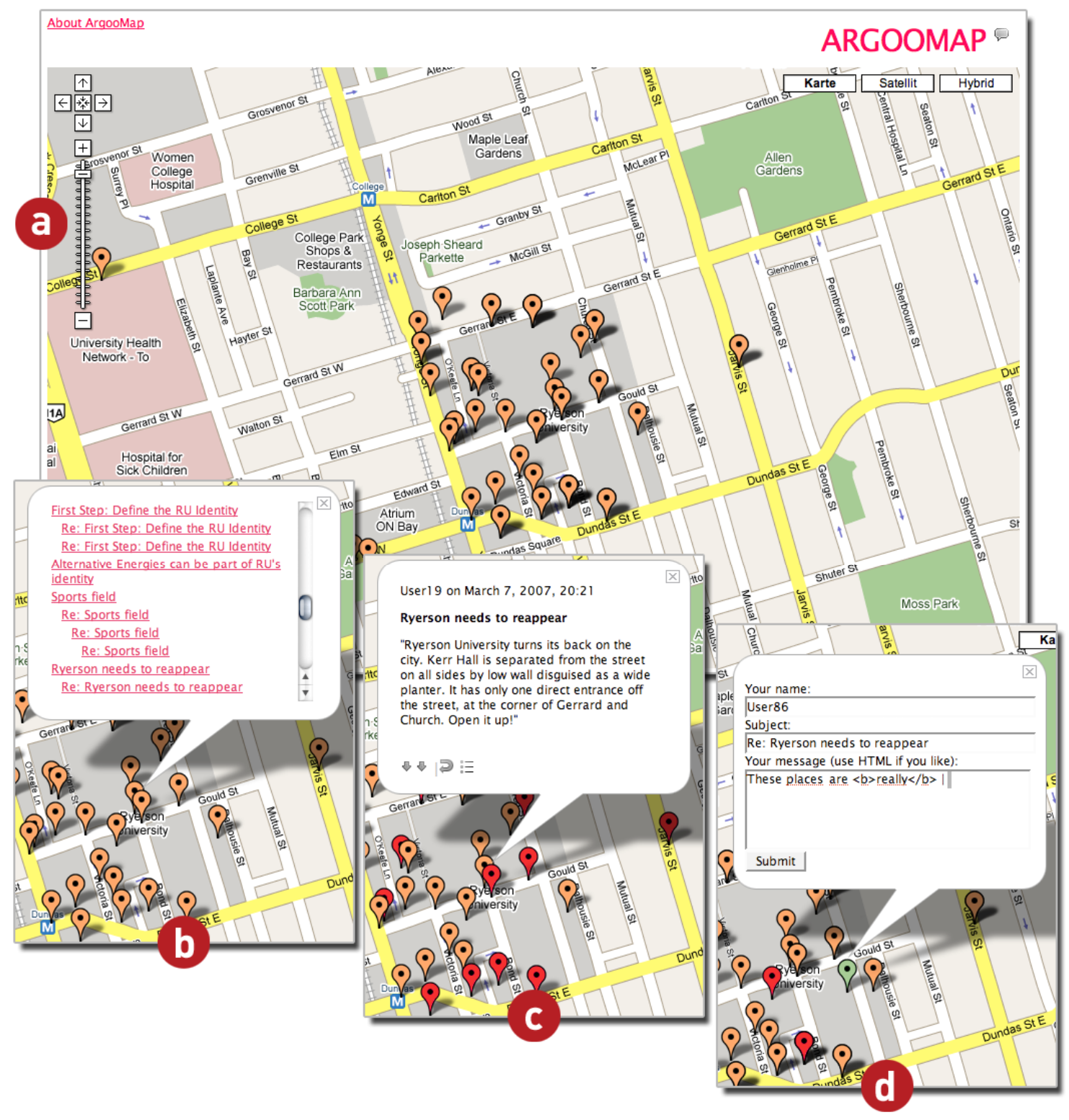

Figure 3: Screenshots of the ArgooMap tool. (a) Initial view with indicators for places that are referenced by contributions to the discussion. (b) Thread-structured overview of the contributions referencing this marker. (c) Display of a single discussion contribution, with the referenced markers highlighted on the map. (d) Input form for a new contribution.

On the technical side, the client is largely based on standard functionality of the Google Maps API. The markers, discussion contributions, and relations between them are stored 
in a server-side MySQL database and pre-processed by a number of PHP scripts in order to be sent in XML format to the client. When adding new contributions and markers to the discussion, the XML data sent by the client is processed accordingly and stored in the database. Through this AJAX-style implementation, the user can continuously interact with the tool without waiting for the reloading of the complete Web page. This overall architecture is outlined in Figure 4.

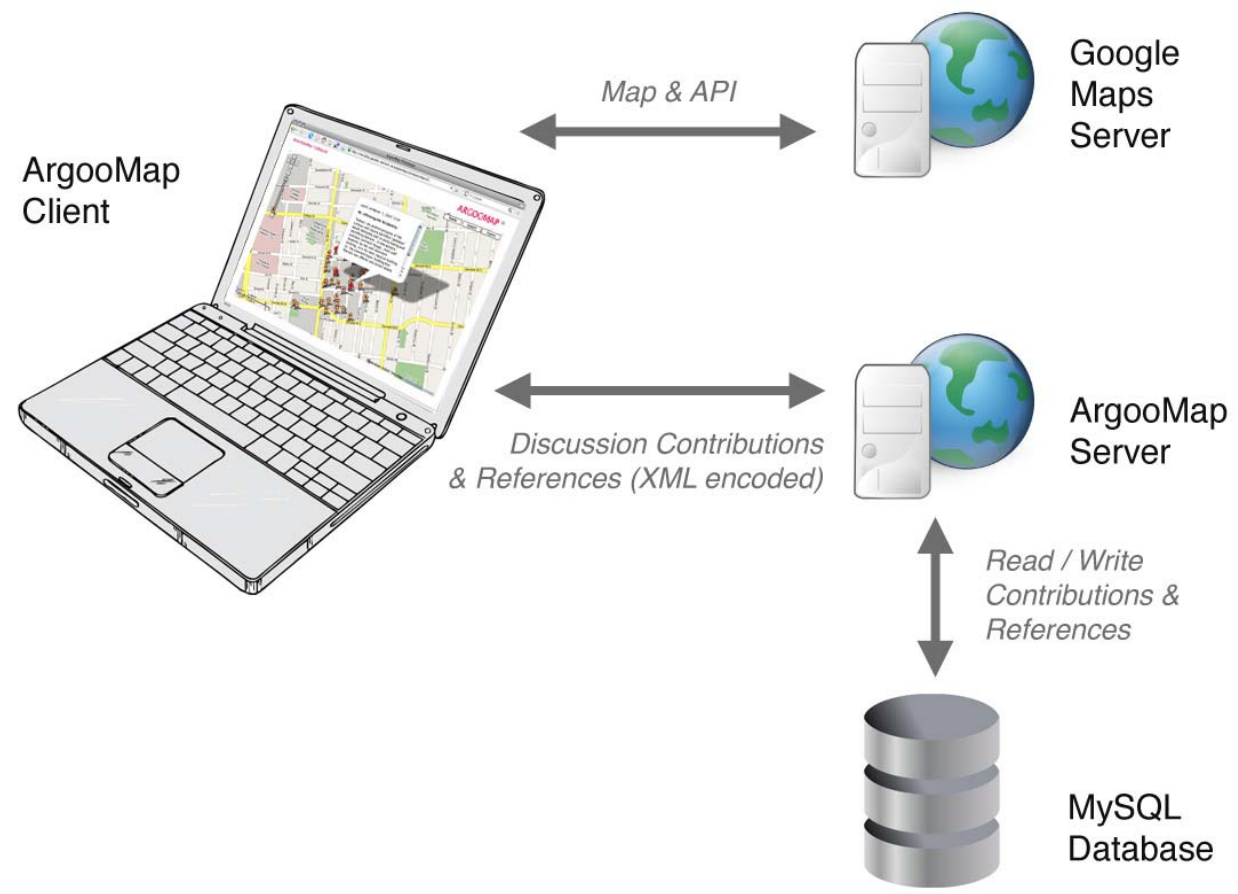

Figure 4: Architecture overview of the ArgooMap prototype.

\section{Mapping Ryerson University's Master Plan Debate}

\subsection{Background and Data Source}

Ryerson University has recently commenced planning a 20-year project aimed at revitalizing the campus and surrounding community in downtown Toronto, Canada. In an attempt to gain an understanding of the visions of students, faculty, and staff members, a discussion was conducted for three days from March 6th to 8th, 2007. The discussion was held in an online forum located on the Ryerson Intranet, to which students and employees 
have access. There were separate discussions for each of the eight major aspects of the master plan including (Ryerson University Master Plan, 2007):

- Reinventing the Heart - Taking a new approach to the heart of the campus: accessible, visible and optimized for University life.

- Reclaiming Streets and Link to Yonge Subway - Integrating downtown streets into the Ryerson campus for maximum connectivity.

- Increasing Close-In Housing Opportunities - Creating living spaces for the Ryerson community.

- Overlapping Campus and City Life - Ryerson's relationship to the City of Toronto.

- An Outreach to Surrounding Community - Ryerson and its neighbourhood: inviting the neighbours in.

- Affirming the RU Identity - Creating a Ryerson identity through visual elements.

These forums were thread-based and no map was provided to participants. Within each forum participants could create new topics or respond to existing threads. Within the thread structure, contributions were ordered by date and time. In each message, the user had the ability to post his/her name or an anonymous ID as well as the subject of the post, and to write his or her thoughts in the message body. Automatically included in every post were the date and the time of the post. The subject line was also pre-filled if the user was responding to an existing thread.

Two discussions were selected for analysis in this study: "Overlapping Campus and City Life" and "Affirming the RU Identity." Table 1 summarizes the participation statistics of the two selected discussions for the Ryerson Master Plan. In the discussion on “Overlapping Campus and City Life”, there were eleven participants who posted a total of 19 contributions, including one starter message posted by the organizers. The average number of contributions per person was 1.72 (median 1). The discussion had three users who posted more than once. There were five new threads started. The average number of replies to a thread was 2.33 (median 2.5). 
In the second discussion that was analyzed, “Affirming the RU Identity”, there were 23 participants who posted a total of 48 contributions with one starter message. The average number of contributions per person was 2.26 (median 1). The discussion had six users who posted more than once (two users contributed 9 and 14 posts). There were nine new threads started. The average number of replies to a thread was 3.25 (median 2).

Table 1: Discussion participation in the Ryerson Master Plan

\begin{tabular}{|l|c|c|}
\hline & $\begin{array}{c}\text { Overlapping Campus } \\
\text { and City Life }\end{array}$ & Affirming the RU Identity \\
\hline Number of participants & 11 & 23 \\
\hline Number of contributions & 19 & 48 \\
\hline Number of contributions per person & $\begin{array}{c}\text { Mean 1.72; Median 1; } \\
\text { Mode 1 }\end{array}$ & $\begin{array}{c}\text { Mean 2.26; Median 1; } \\
\text { Mode 1 }\end{array}$ \\
\hline Number of new threads started & 5 & 9 \\
\hline Total number of replies & 14 & 39 \\
Order of replies & 3 & 11 \\
$1^{\text {st }}$ Order & 3 & 9 \\
$2^{\text {nd }}$ Order & 4 & 4 \\
$3^{\text {rd }}$ Order & 2 & 3 \\
$4^{\text {th }}$ Order & 1 & 3 \\
$5^{\text {th }}$ Order & 1 & 2 \\
$6^{\text {th }}$ Order & - & 2 \\
$7^{\text {th }}$ Order & - & 1 \\
$8^{\text {th }}$ Order & - & 1 \\
$9^{\text {th }}$ Order & - & 1 \\
$10^{\text {th }}$ Order & - & 1 \\
$11^{\text {th }}$ Order & - & 1 \\
$12^{\text {th }}$ Order & Mean 2.33; Median 2.5; & Mean 3.25; Median 2; \\
\hline Number of replies to threads & Mode 3 & Mode 1 \\
\hline Contributions on day 1 & 5 & 10 \\
\hline Contributions on day 2 & 10 & 26 \\
\hline Contributions on day 3 & 4 & 12 \\
\hline
\end{tabular}

\subsection{Simulation Method}

The simulation exercise kept the existing thread structure of the discussion, but each message was given one or more explicit geographic references. We then copied the two discussions into the ArgooMap tool in order to demonstrate how participants could have benefited from the use of a Web 2.0-based online mapping component.

The study defines three levels of detail for geographic references: high, medium, and low. A high level of detail is found at a large map scale where individual objects can be 
clearly identified and marked using a point. This includes building entrances, specific street corners, or even signs on campus. Medium level of detail describes references such as an entire building or a section of a street. Finally, a low level of detail occurs when the entire university campus or a street (name) with no specific location is referenced. The purpose of defining these levels of detail is to highlight the value of the explicit geographic references in map-based discussions. If low-level geographic references are predominant, then the usefulness of such an application might be limited. In contrast, if there are more medium- and high-level references, the usefulness of the application might be greater due to the ability to accurately reference locations of interest.

The geographic references were determined from the contents of each message. For example, one contributor writes, "Kerr Hall needs to be opened up”. The associated geographic reference to this message is 'Kerr Hall' and as a result a marker would be placed in the centre of this building and the message attached to it. To deal with lowdetail geographic reference such as a street name, the marker was placed at the corresponding label in Google's "map” view, which was most central in the study area. For example, where a contributor is discussing the university as a whole, a marker will be placed on the 'Ryerson Polytechnic University' label. In many instances, messages contained more than one geographic reference. In such situations, ArgooMap allows for a message to be associated with several markers. The context of the debate is the Ryerson Master Plan, so it can be assumed that virtually all messages are related to the university as a whole. For this reason every contribution was referenced to Ryerson University even in situations where there was no such reference stated (classified as an implied reference).

For the purpose of this study, two separate maps were created, one for each discussion forum. We were interested in differences between the spatial patterns of geographic references between the discussions as one had a more geographic subject (campus/city) than the other (Ryerson identity). The number of geographic references found in the thread discussion was used to understand if there is value in using an application such as ArgooMap in planning debates. Information including the number of posts with geographic references, the number of posts with more than one geographic reference, the 
number of unique references, and the level of detail for each geographic reference, were extracted and analyzed to understand the utility of the application.

\subsection{Geographic References in Original Debate}

Table 2 summarizes the geographic reference statistics extracted from the thread-based discussion. In the discussion on “Overlapping Campus and City Life”, all 19 contributions contained a geographic reference with 13 having multiple references. There were 21 unique geographic references made, and they were used 56 times throughout the discussion. The most frequent references (besides Ryerson University, which was referenced in all contributions) were 'Dundas Square' and 'Yonge Street', each being mentioned six times. The average number of geographic references per post was 2.94 (median 3). From the 11 participants contributing to this discussion, each person referenced on average 1.91 (median 1) unique geographic locations. Table 2 further highlights the proportions of geographic detail found in the references extracted from the discussion. From the 21 geographic references made, five were low detail references (cited 31 times), 16 were medium detail references (cited 25 times), and there were no high detail references made.

In the discussion on "Affirming the RU Identity”, 47 of the 48 contributions contained a geographic reference with 31 containing multiple references. In the one message without a reference, Ryerson University was the subject so that the message was given an implied reference. There were 54 unique geographic references made, and they were used 150 times throughout the discussion. The most frequent reference (besides Ryerson University) was the 'City of Toronto’ mentioned six times. Further, 16 references were made to different locations at the University of Toronto. The average number of geographic references per post was 3.13 (median 2). On average, there were 2.30 (median 1) unique geographic locations referenced per person. The proportions of geographic detail found in the references extracted from the discussion include 17 low detail references (cited 88 times), 32 were medium detail references (cited 56 times) and five were high detail references (cited six times). 
Table 2: Geographic references statistics

\begin{tabular}{|l|c|c|}
\hline & $\begin{array}{c}\text { Overlapping Campus } \\
\text { and City Life }\end{array}$ & $\begin{array}{c}\text { Affirming the RU } \\
\text { Identity }\end{array}$ \\
\hline Contributions with a geographic reference & $19(100 \%)$ & $48(100 \%)$ \\
\hline Contributions with multiple geographic references & $13(68 \%)$ & $31(65 \%)$ \\
\hline Implied geographic references & 0 & 1 \\
\hline Number of unique geographic references & 21 & 54 \\
\hline Total number of geographic references made & 56 & 150 \\
\hline Number of geographic references per contribution & $\begin{array}{c}\text { Average 2.94; } \\
\text { Median 3; } \\
\text { Mode 2 }\end{array}$ & $\begin{array}{c}\text { Average 3.13; Median } \\
2 ; \\
\text { Mode 1 }\end{array}$ \\
\hline $\begin{array}{l}\text { Number of unique geographic references contributed per } \\
\text { participant }\end{array}$ & $\begin{array}{c}\text { Average 1.91; } \\
\text { Median 1; } \\
\text { Mode 1 }\end{array}$ & $\begin{array}{c}\text { Average 2.30; } \\
\text { Median 1; } \\
\text { Mode 0 0 }\end{array}$ \\
\hline Number of unique low detail geographic references & 5 (23.8\%) & 17 (31.4\%) \\
\hline Number of unique medium detail geographic references & 16 (76.2\%) & 32 (59.3\%) \\
\hline Number of unique high detail geographic references & 0 & 5 (9.3\%) \\
\hline Number of times low detail geographic references were made & 31 (55.4\%) & 88 (58.6\%) \\
\hline $\begin{array}{l}\text { Number of times medium detail geographic references were } \\
\text { made }\end{array}$ & 25 (44.6\%) & 56 (37.3\%) \\
\hline Number of times high detail geographic references were made & 0 & $6(4 \%)$ \\
\hline Most frequent geographic reference & $\begin{array}{c}\text { Dundas Square, Yonge } \\
\text { Street }\end{array}$ & City of Toronto \\
\hline
\end{tabular}

\section{Case Study Results and Discussion}

\subsection{General Effects of Simulation Exercise}

The simulation highlighted some interesting characteristics that were not obvious in the thread-based discussion. First, both discussions were very geographic in nature, with almost all of the contributions having at least one geographic reference, averages around three geographic references per post, and between two and three unique references per person. These values suggest that there is great potential for argumentation mapping in planning debates.

The most common type of unique geographic reference used was of medium detail. At this level of detail, there were 16 (76.2\%) unique references made in the "Overlapping Campus and City Life" discussion and 32 (59.3\%) in the "Affirming the RU Identity" discussion. This shows that people were willing to discuss topics at a relatively specific 
level of detail. The reason for not using more specific geographic references could be attributed to the effort in describing such locations in words. Had this discussion been run initially with a mapping component, the ease of placing a marker at a very specific location might have spurred the use of higher levels of detail in geographic references.

Geographic references with a low level of detail, however, were the most frequently referenced at 31 (55.4\%) of the total number of references in one discussion and 88 (58.6\%) in the other. These numbers are high because of the frequency of referencing Ryerson University. Also, the amount of low detail geographic references could be associated with the ease of description. Describing low level references such as the "University of Toronto" is much easier to understand than a high level reference such as "the grad residence with the huge U of T sign".

The ease of placing reference markers for different levels of detail depended upon which of the three different Google Maps views was activated. For low level of detail references, the "map" view was found to be the easiest to use. Only landmark buildings, parks, and institutions such as hospitals are shown in this view. The Ryerson University and the University of Toronto are identified as well. Placing medium and high detail geographic references seemed easier in the "satellite" or "hybrid" view with their increased surface detail. It was possible to identify individual buildings and other objects on the ground and place a marker there.

\subsection{Links between Geographic References and Thread Structure}

Figure 5 shows how the implicit reference to Ryerson University in all messages was used to preserve the original thread structure of the Master Plan debate in the simulated discussion within ArgooMap. The highlighting of reference markers in ArgooMap makes it easy to identify existing geo-argumentative relations (Rinner 2006). A geospatial relation between two messages exists when they reference the same location, or two locations that are in a geospatial relation (such as two neighbouring buildings). An argumentative relation between two locations exists when they are referenced by the 
same contribution, or when they are referenced by two contributions that are argumentatively related (such as replying to one another).

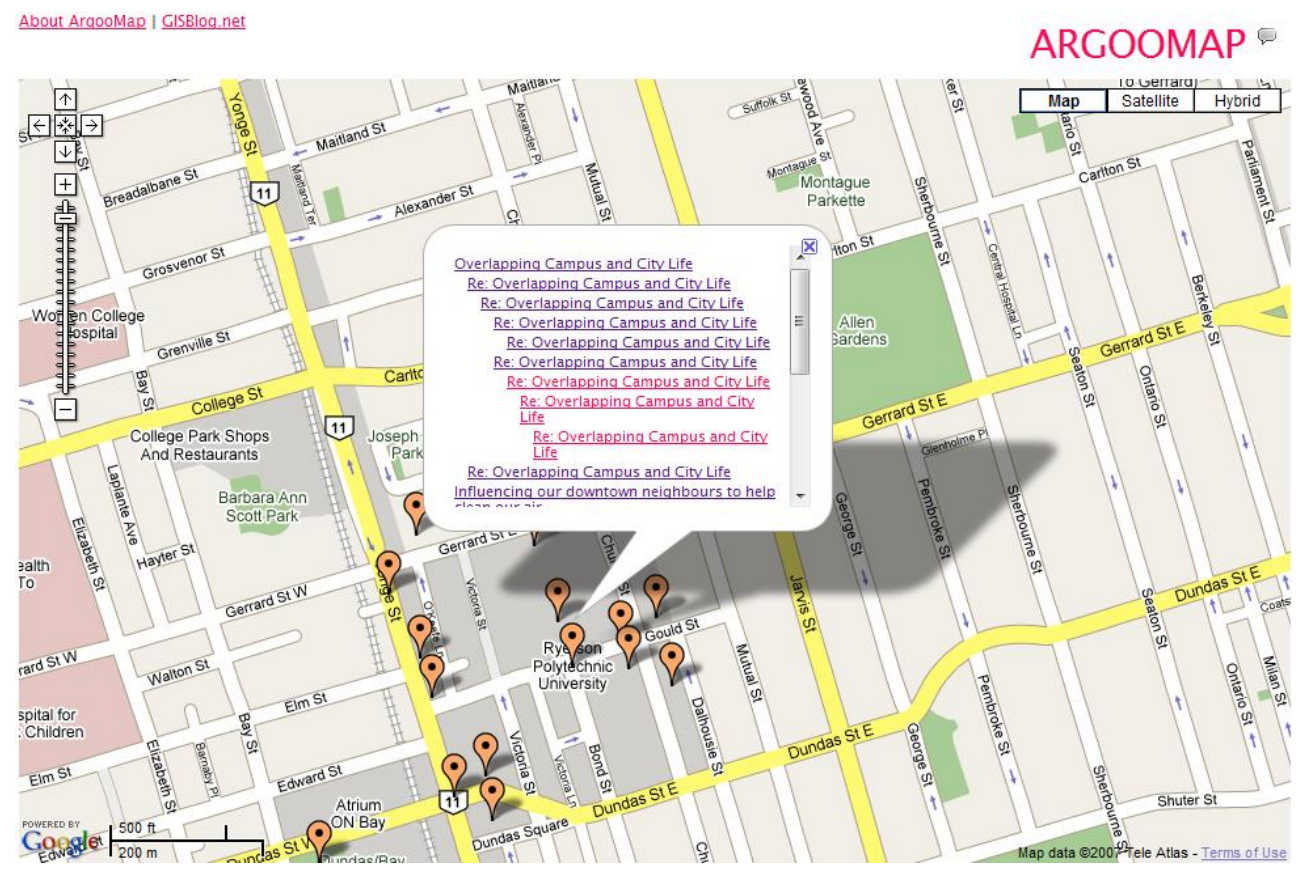

Figure 5: Preservation of thread structure in map-based discussion forum through implied reference to Ryerson University.

An example from the study is shown in Figure 6 and Figure 7. Figure 6 shows the initial message 1, which includes nine geographic references (italicized). It reads as follows:

"Architecture - buildings that standout. $R B B$ is new, but essentially blends in with $B B$ and Canadian Tire. It could be argued that those retail chains have more of a presence along Dundas. Look at $U$ of $T$ with their new and distinct buildings, the Bayhern (sp?) Centre, MaRS, the new pharmacy building with its suspended POD-like lecture room. Also, notice how those buildings use a lot of glass to really stand out and maximize the use of natural light. Ryerson has Kerr Hall, which is unique in its Quad design. Even $U$ of T's bookstore is pretty unique.

Does Ryerson have any sort of 'gateway' structure that says, welcome to Ryerson, or that you're now entering the Ryerson campus?” 
The geographic references in this text include 'Dundas Street', 'University of Toronto', 'Canadian Tire', and 'Kerr Hall'. These locations have a direct argumentative relation to Ryerson University because they are related through the text of message 1.

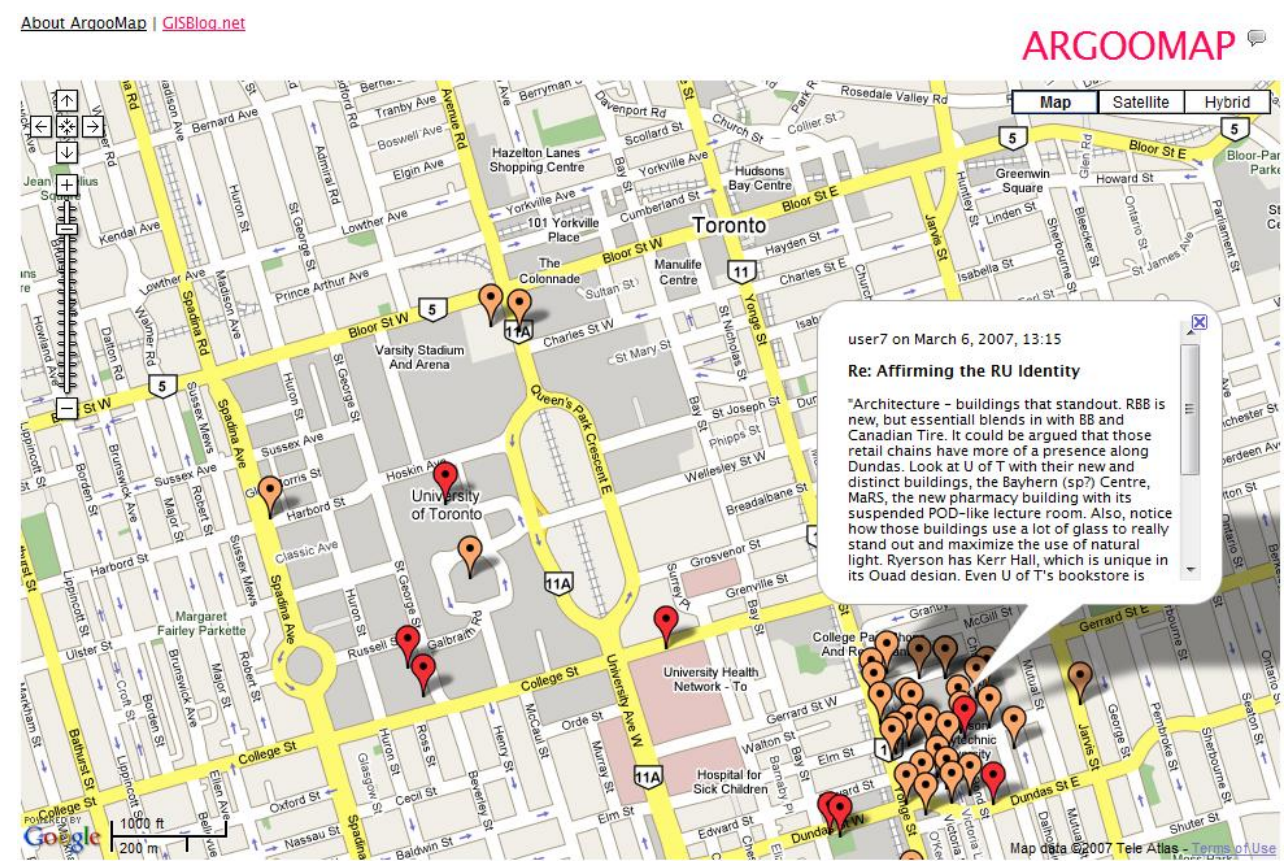

Figure 6: Geographic references associated with selected message 1

Figure 7 shows message 2, which shares a common geographic reference with message 1 . It also is a 4th-order reply to message 1 and reads as follows:

"I agree, maybe there could be a main artery or loop that branches out to the main buildings on campus. This would allow a person to follow a path to see all or most of campus or just makes it easier to give directions. $U$ of $T$ has King's College Circle, which is a real strong focal point for the campus."

The shared geographic reference is the 'University of Toronto'. Message 1 and message 2 become directly related through their geographic references in the argumentation map, although they are only in a 4th-order argumentative relation through the thread structure. Similarly, a message and its direct reply would create an argumentative relation between two initially unrelated locations. 


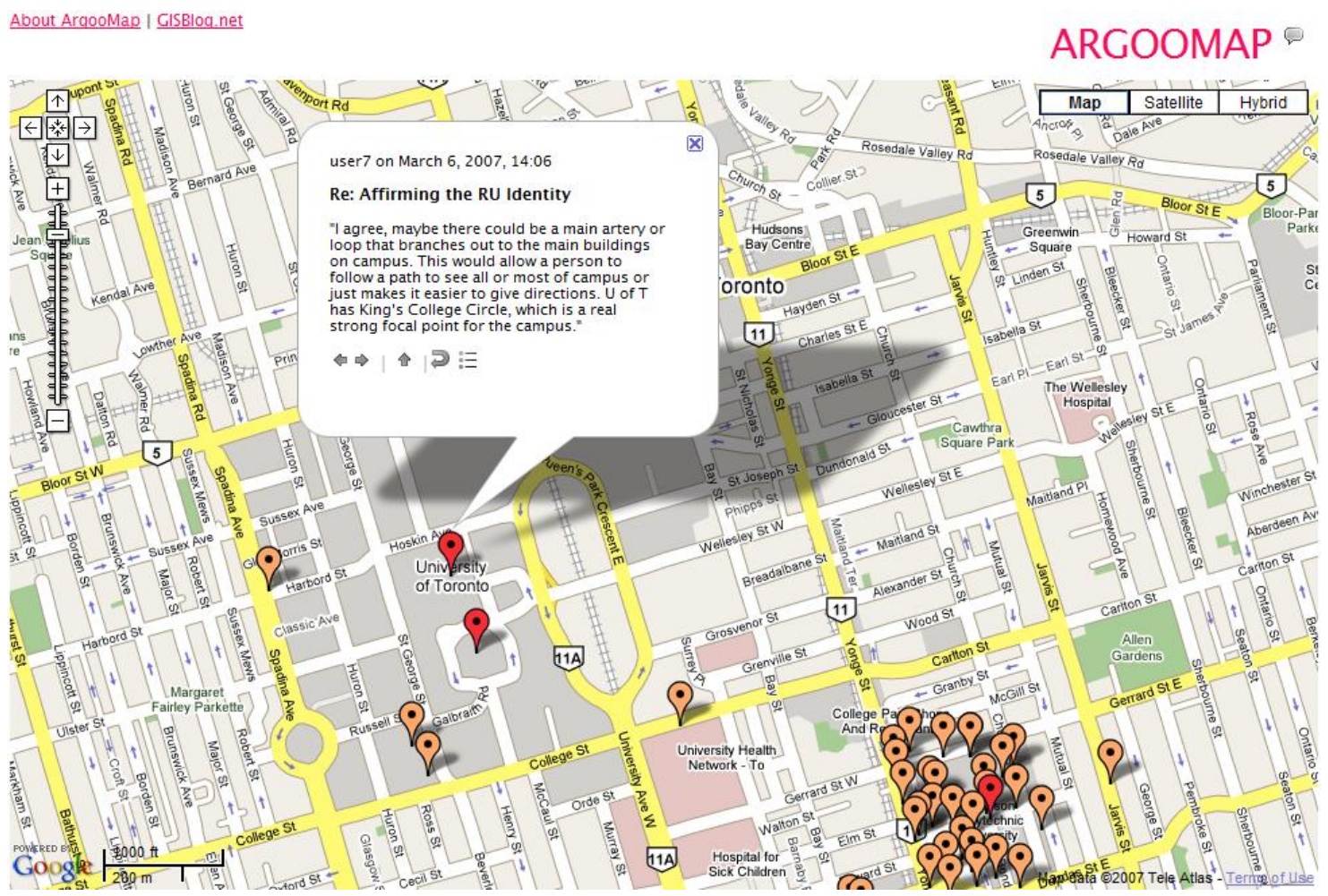

Figure 7: Message 2 provides an example of a geo-argumentative relation - a relation between two discussion contributions through their common geographic reference

\subsection{Benefits of Online Map-Based Discussion Forum}

The extracted geographic references from the Master Plan debate demonstrate the potential to clarify the participants' arguments in planning discourses and support a better understanding of what is being discussed in each contribution. This is especially true for references of medium and high level of detail. In addition, areas of varying degrees of interest can be identified through the clustering of references. Looking at the markers for one of the two forums on a small scale map, an expected cluster of markers exists around the Ryerson University campus. On a larger scale map, a cluster of markers can be seen on the west side of Ryerson's Campus. This latter trend might suggest that attention should be focused on the campus and more specifically on its west side in order to achieve the goal of "Affirming the RU Identity". 
Understanding geo-argumentative relations in a discussion can provide useful insight into the spatial thought process of the participants. In the example above it was suggested by a participant that the University of Toronto has a strong identity as a result of their new buildings. The participant goes on to suggest that a 'gateway structure' would help welcome people to Ryerson University and as a result strengthen its identity. In this respect, the map-based discussion forum goes beyond geotagged Internet forums, such as the Google Earth Community forums at http://bbs.keyhole.com. In the latter, individual messages can be localized on Google Maps or in Google Earth but the centre of discussion remains the threaded forum. In contrast, map-centred argumentation allows for bi-directional, m:n linkages between messages and places and therefore enables both, forum-based or map-based viewing and participation.

Finally, since the ArgooMap tool is based on the Google Maps API, it provides an interface that many Internet users are already comfortable with. There are few functions that a user would not know from Google Maps. From a developer's point of view, customizing Google Maps is inexpensive and provides access to free geographic data.

\subsection{Limitations}

During the simulation exercise, some limitations of the ArgooMap prototype became apparent. Ensuring the right scale was used for placing geographic references was very important. Placing and viewing the markers at different scales can produce different results or interpretations of the references. As seen in Figure 8 it becomes increasingly difficult to distinguish between markers on small map scales. The value of the detailed geographic references such as the ' $\mathrm{U}$ of T Sign' becomes lost when zoomed out to this scale. However references such as the 'City of Toronto' or 'Mississauga' become more meaningful because such locations are commonly referenced by a single point at this scale. An additional challenge was the dynamic label placement in Google Maps. At different scales, the labels appear at different locations or appear/disappear. In this study the location of map labels at the second Google Maps zoom level was used. 
Another limitation of the Google Maps API was that the text window containing the contributor's comments might cover and hide a portion of the map. It would be beneficial if the location of the text windows could be modified by the user. Further, it is impossible to view more than one text window at a time. Having this ability would assist in studying the flow of discussion and allow users to view responses attached to different markers.

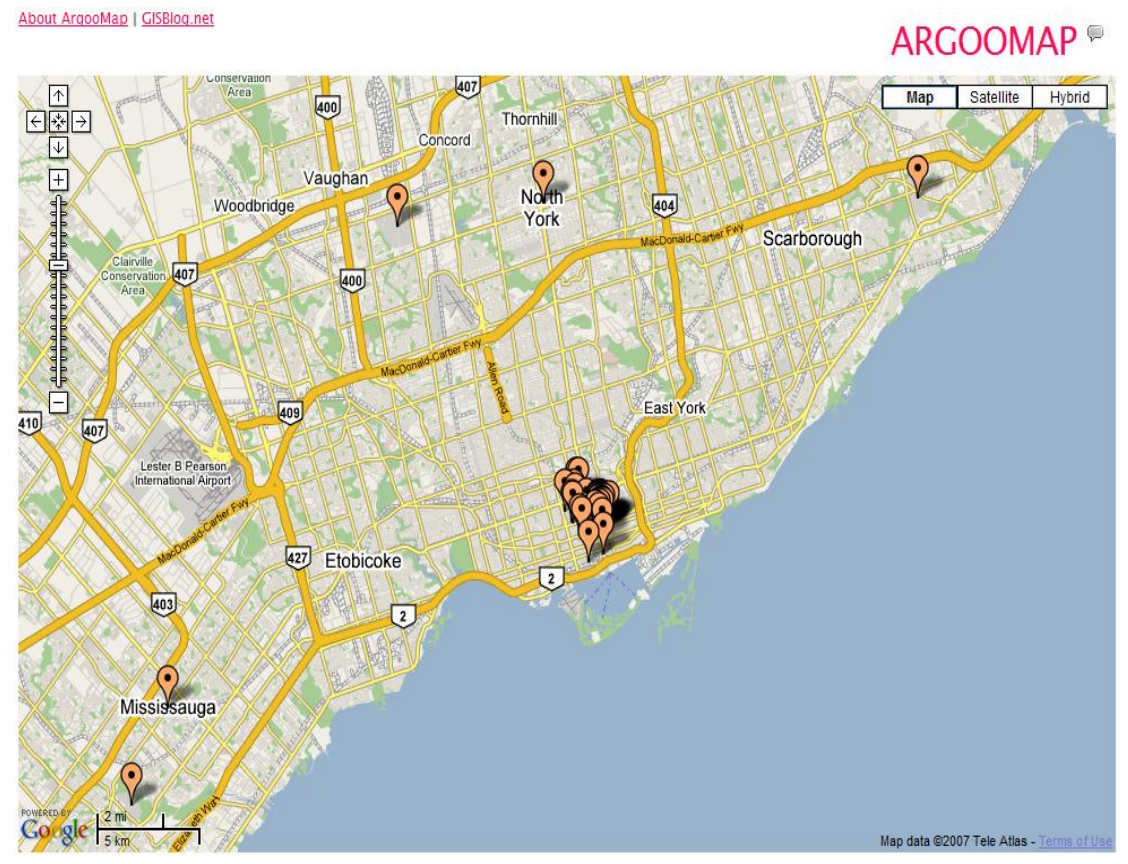

Figure 8: ArgooMap showing markers at a small map scale

\section{Conclusions and Outlook}

In this paper, we discussed the use of Web 2.0 concepts and technology to support the deliberative aspects of spatial decision-making processes. A map-based discussion forum was presented that is built on the Google Maps platform and enables Internet users to submit place-based comments and respond to other participants' contributions. Using a simulated planning debate, we could show how an argumentation map can be used to gain an overview of the status of a debate and help understand the participants' spatial thought processes by navigating the network of messages and geographic references. 
In order to move from discussion support to decision support, the ArgooMap tool could be extended by a voting method for participants to express their preferences about places without having to contribute messages. For example, a voting system would allow for a quick way to see how many users ‘agree' or 'disagree' with a certain message or geographic location. Further, such functionality could give the facilitators a quick indication of which messages or topics are important to the participant group (through the volume of voting), and which messages or topics are supported/accepted (through results of the voting).

The geographic references in this tool are linked to entire messages, not to geographic identifiers found in the texts. Highlighting each geographic names within messages would create an even more explicit link between messages and map. This would provide greater clarification of the participants' statements and reduce the need for interpretation by another participant. A related development concerns the automatic, real-time extraction of geographic references from contributions through a gazetteer service and thus, automatic or semi-automatic spatial referencing.

We are currently preparing a realistic case study using an improved version of ArgooMap. Another goal is to devise standardized evaluation methods to assess the benefits and limitations of Web 2.0-based PGIS, and promote their use in planning and public policy. Ultimately, we seek to close the gap between GIS concepts, methods, and tools, and the ad-hoc development of geospatial Web 2.0 technologies and applications.

\section{Acknowledgements}

Sepehr Mavedati assisted with the development and revision of the ArgooMap tool. Helpful input on this research was received from Soheil Boroushaki. Ashley Korol and Jyothi Kumari provided numerous suggestions to improve the manuscript. This research was partially funded through the German Academic Exchange Services (DAAD), the Canadian GEOIDE Network of Centres of Excellence, and the Natural Sciences and Engineering Research Council of Canada (NSERC). 


\section{References}

deegree (2007) deegree - Free Software for Spatial Data Infrastructures. Web site available at http://www.deegree.org [last visited January 14, 2008]

GeoRSS (2008) GeoRSS - Geographically Encoded Objects for RSS feeds. Web site available at http://georss.org/ [last visited January 14, 2008]

Goodchild, M.F. (2007a) Geographic Information Science: The Grand Challenges. In J. Wilson, A.S. Fotheringham: The Handbook of Geographic Information Science. Malden, MA: Blackwell Publishing, pp. 596-608

Goodchild, M.F. (2007b) Citizens as Voluntary Sensors: Spatial Data Infrastructure in the World of Web 2.0. International Journal of Spatial Data Infrastructures Research 2: 2432

Google (2008) Google Maps API. Web site available at http://code.google.com/apis/maps/ [last visited January 14, 2008]

Gore, A. (1998) The Digital Earth: Understanding our Planet in the 21st Century. Lecture given at the California Science Center, Los Angeles, California, on January 31, 1998.

Available online at http://www.digitalearth.gov/VP19980131.html and http://www.isde5.org/al_gore_speech.htm [last visited January 4, 2008]

Graham, P. (2005) Web 2.0. Essay dated November 2005. Available online at http://www.paulgraham.com/web20.html [last visited January 4, 2008]

O'Reilly, T. (2005) What Is Web 2.0? Design Patterns and Business Models for the Next Generation of Software. Essay dated 09/30/2005. Available online at http://www.oreillynet.com/lpt/a/6228 and 
http://www.oreillynet.com/pub/a/oreilly/tim/news/2005/09/30/what-is-web-20.html [last visited January 4, 2008]

Harris, T.M., Weiner, D., Warner, T.A., and Levin, R.(1995) Pursuing social goals through participatory geographic information systems. Redressing South Africa's historical political ecology. In Pickles, J. (ed.): Ground Truth: The Social Implications of Geographic Information Systems. New York: Guilford Press, pp. 196-222

Jankowski, P., and Nyerges, T. (2003) Toward a Framework for Research on Geographic Information-Supported Participatory Decision-Making. URISA Journal 15(APA I): 9-17

Keßler, C., C. Rinner, and M. Raubal (2005a). An Argumentation Map Prototype to Support Decision-Making in Spatial Planning. 8th AGILE Conference, Estoril, Portugal.

Keßler, C., M. Wilde, and M. Raubal. (2005b). Using SDI-based Public Participation for Conflict Resolution. Proceedings of the 11th EC-GI \& GIS Workshop (CD-ROM), Alghero, Sardinia.

Map Builder (2008) Map Builder - Rapid mashup development tool for Google and Yahoo maps. Web site available at http://www.mapbuilder.net/ [last visited January 14, 2008]

MapServer (2008) Mapserver - Open Source development environment for building spatially-enabled internet applications. Web site available at http://mapserver.gis.umn.edu/ [last visited January 14, 2008]

Microformats (2008) Microformats. Web site available at http://www.microformats.org [last visited January 14, 2008] 
Nyerges, T, Barndt, M., and Brooks, K. (1997) Public Participation Geographic Information Systems. Proceedings of Auto-Carto 13, Seattle, WA, American Congress on Surveying and Mapping, Bethesda, MD, pp. 224-233

OpenStreetMap (2008) OpenStreetMap, The Free Wiki World Map. Web site available at http://www.openstreetmap.org/ [last visited January 14, 2008]

Renn, O. (1999) A Model for an Analytic-Deliberative Process in Risk Management. Environmental Science \& Technology 33(18): 3049-3055

Rinner, C. (2001) Argumentation Maps - GIS-based Discussion Support for Online Planning. Environment and Planning B: Planning and Design 28(6): 847-863

Rinner, C. (2006) Argumentation Mapping in Collaborative Spatial Decision Making. In S. Dragicevic, S. Balram (Eds.): Collaborative GIS. Idea Group Publishing, pp. 85-102

Rinner, C., Bird, M. (forthcoming) Evaluating Community Engagement through Argumentation Maps - A Public Participation GIS Case Study. Environment and Planning B: Planning and Design

Sidlar, C., Rinner, C. (2007) Analyzing the Usability of an Argumentation Map as a Participatory Spatial Decision Support Tool. URISA Journal 19(1): 47-55

Simao, A., Densham, P.J., Haklay, M. (forthcoming) Web-based GIS for Collaborative Planning and Public Participation: An Application to the Strategic Planning of Wind Farm Sites. Journal of Environmental Management

Stern, P.C., Fineberg, H.V. (1996) Understanding Risk: Informing Decisions in a Democratic Society. National Academy Press 
Twittervision (2007) Twittervision by David Troy. Web site available at http://twittervision.com/ [last visited January 14, 2008]

WikiMapia (2008) WikiMapia - Let's describe the whole world. Web site available at http://wikimapia.org/ [last visited January 14, 2008] 